\title{
Pasture API: A digital platform to support grazing management for southern Australia
}

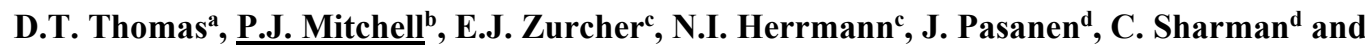 \\ D.A. Henry ${ }^{\mathrm{e}}$ \\ ${ }^{a}$ CSIRO Agriculture and Food, Floreat, Western Australia. \\ ${ }^{b}$ CSIRO Agriculture and Food, Hobart, Tasmania. \\ ${ }^{c}$ CSIRO Agriculture and Food, Canberra, Australian Capital Territory. \\ ${ }^{d}$ CSIRO Data 61, Hobart, Tasmania. \\ ${ }^{e}$ CSIRO Food Innovation Centre, Werribee, Victoria. \\ Email:dean.thomas@csiro.au
}

\begin{abstract}
Extensive ruminant livestock production is complex, and it is difficult and time-consuming to obtain quantitative information for decision making (e.g. biomass and quality of forage). Increasingly data from historic records, seasonal forecasts, or near real-time data from remote or on-farm sensors. However, there is limited capacity to compile, integrate and analyse these data. In some cases, there are decision support tools available with such analytical capability, but the time required to learn and apply these to gain the benefits is significant, and a major disincentive to their widespread adoption. Based on the need for simple, entry level, decision support for the livestock industry we have developed the Pasture API (Application Programming Interface) platform. The aim of the Pasture API was to build a seasonal pasture forecast system that can provide a forecast to specific locations up to 6 months into the future for anywhere in Australia and made available to a variety of client software packages. The platform is able to forecast a wide range of simulated data outputs including pasture biomass, ground cover, supplementary feeding and livestock growth.
\end{abstract}

The Pasture API application required the integration of a number of both new and existing analytical capabilities, which are summarised below and described in more detail in this paper.

1) Ruminant grazing simulation engine: The GrazPlan biophysical pasture and ruminant nutrition model (adapted from GrassGro ${ }^{\mathrm{TM}}$ software) was repurposed for use as the modelling engine in a backend service infrastructure.

2) Flexible tactical grazing scenarios: The GrazPlan models were incorporated into a new software application for batch processing of tactical grazing scenarios. This application was called GGTactical.

3) Dynamic platform for connecting the pasture simulation engine with data streams: We used CSIRO's Senaps platform to connect the GGTactical application with a range of spatiotemporal data streams so that simulation scenario workflows could be implemented.

4) Demonstration interface: A demonstration website (Pasture Tracker) was built to interact with the Pasture API application and implement workflow's based on location and livestock enterprise details. Currently the software is hosted internally by CSIRO, with the intention that a version become publicly available in the near future.

We demonstrated that Pasture API is able to replicate simulation of a livestock grazing scenario, as can be done with more complex modelling software, such as GrassGro ${ }^{\mathrm{TM}}$. Key production metrics such as net primary productivity (NPP) of pasture, supplementary feeding, ground cover and liveweight of stock were charted. These were reported both as historic percentile values across a season, the now-cast (current) value, and a probabilistic forecast for a predefined period of time (e.g. 3 or 6 months).

To compare the effects on forecasts for various input data streams that were available, a sensitivity analyses was conducted. This provided information about the suitability of more generic data streams (e.g. national soils database) for forecasting, comparing forecast outcomes for those where local data were available.

The Pasture API project demonstrates the ability to create easy to use, yet powerful, decision support systems for the livestock industry. This is a novel integrating technology that we expect to continue to develop to make use of the many sources of sensor and archive data that are collected within livestock businesses. This information is expected to increase the precision across a range of interventions, including; stocking density, timing of paddock rotations, and supplementary feeding. In the future we expect to increase the use of local data streams and refine the data delivery processes to produce the sitespecific information sought by the industry for decision making.

Keywords: Simulation modelling, ruminant nutrition, extensive grazing systems, Senaps 


\section{INTRODUCTION}

Extensive livestock production is important to the agricultural industry globally and makes a substantial contribution to the livelihood of local communities. Livestock produced on extensive agricultural lands rely primarily on the forage that is grown to meet their nutrient requirements. To meet the seasonal feed demands of their animals, livestock managers often use a range of forage sources (such as pastures, crop residues, forage crops and supplementary feeding), which is referred to as the feedbase (Bell et al. 2009). Both the amount and quality (energy density) of feedbase components varies according to the growth cycles of the forage plants and the climatic conditions under which they grow. To manage livestock in these systems, livestock producers rely on a wide range of skills and information to deal with the high within and between-season variability in the feedbase.

To assist in managing the complex nature of the feedbase, researchers have developed a range of information and decision support systems. It is not the intention of our paper to review these systems (see Bell et al. 2009), however it is worth pointing out some of the existing limitations and how we propose that these might be addressed.

Sensor data: Monitoring soils, vegetation and livestock in ruminant grazing systems is limited by capacity to integrate and project forward the associated outcomes and production pathways. While sensor data has in some cases been informative, and well utilised (e.g. remotes sensing of pasture growth using Pastures from Space ${ }^{\mathrm{TM}}$ (Mata et al. 2004)), the integration of this information for farm decision making has relied on the knowledge and experience of the livestock producer. In many cases, some quantitative data is used as a basis to estimate future outcomes with a high degree of subjectivity.

Meteorological and other environmental databases: A network of weather stations across Australia provides an important resource for the agricultural industry, both in terms of in-season information but also a historical archive of meteorological records over many decades. The development of gridded soils information e.g. the "getApsoil" web service (http://www.asris.csiro.au/ASRISApi/api/APSIM/getApsoil) provided by the Australian Soil Resource Information System (ASRIS) can support the broad-scale application of predictive agricultural tools across large spatial domains. Accurately characterising pasture composition across the diverse agro-ecological regions of southern Australia remains a significant challenge. To enable a reasonable approximation of pasture species composition, a plant model library of geo-referenced pasture vegetation has been built to represent typical pastures grown for livestock production in the project regions. This was based on information collected at Statistical Local Area (SLA) resolution that was described in the MLA Feedbase Audit (Donald, 2012). Access and efficient processing of these data is needed to support tools that use historic climate information to predict probabilistic outcomes.

Agricultural simulation modelling: A number of biophysical simulation modelling platforms have been developed for agriculture by Australian researchers. However, the complexity of these models and some inflexibility with their deployment presents an ongoing challenge to their uptake and utilisation. Some of the required processes such as model parameterisation, and sourcing/implementing input data can be highly technical and tedious. This is particularly the case when a large number of scenarios and locations need to be considered, which is generally the case due to the variability in climate, soils and farming enterprises.

Pasture API is a project funded by the CSIRO that aims to provide a platform to bring together sources of spatiotemporal input data for modelling extensive livestock grazing systems of southern Australia, to help address many of the constraints outlined above. Specifically, we focus on the implementation of a platform to increase simplicity and efficiency of use of the GrazPlan ruminant grazing models (Moore et al. 1997). This paper describes the processes that were put in place to produce a digital platform that enables ruminant livestock production to be simulated and outcomes forecast for any chosen location across southern Australia. Our intention initially was to develop a generic platform, to enable access to coarse data with a national footprint (e.g. climate, soils and vegetation). However, in future we see a role for improving the resolution of input data from local data streams such as remote and proximal sensors, soil sampling, pasture composition and characteristics of a specified livestock enterprise. Therefore, a key part of this study was to conduct a sensitivity analysis to evaluate any benefits from either improving the resolution of input data from local data sources or to determine the implications for forecasting when the input data used is less accurate.

\section{MODELLING PROCESSES}

\subsection{Simulation modelling and livestock model parameterization}

The project used the GrazPlan Pasture and Ruminant models (Moore et al. 1997) for simulating livestock grazing scenarios, implemented in the GrassGro ${ }^{\mathrm{TM}}$ software application. These simulations are run on a daily 
time step, and we used the tactical modelling function to simulate probabilistic outcomes over a selected forecast period based on 30 years of historical data; 1 January 1989 to 31 December 2018. The livestock enterprises used in the project were stored in GrassGro ${ }^{\mathrm{TM}}$ library files and included a range of sheep and cattle enterprises. Similarly, a GrassGro ${ }^{\mathrm{TM}}$ library was used to store a range of different pasture types representing typical pastures grown for livestock production in the project regions. The modelled pasture types were selected from the existing GrassGro ${ }^{\mathrm{TM}}$ pasture species, either based on i) local consultation with industry people or ii) matching pasture species described in the MLA Feedbase Audit (Donald, 2012) with GrassGro ${ }^{\mathrm{TM}}$ pasture species for Statistical Local Areas (SLA), as described by Thomas et al. (2019). Pastures in the GrassGro ${ }^{\mathrm{TM}}$ library were deployed to the required simulation scenario using processes described by Herrmann and Zurcher (2011).

Input data were assigned to simulations either through association with a particular location or by searching by georeference. Meteorological data were obtained from SILO gridded datasets for maximum and minimum temperature, rainfall, synthetic pan evaporation, radiation, and vapour pressure (Jeffrey et al. 2001). Data for the SILO grid point (resolution of 0.05 degree) nearest the fish-net grid point was transformed into APSIM format for use in the simulations. Soil data_for the GrassGro ${ }^{\mathrm{TM}}$ simulations were obtained via the "getApsoil" web service (http://www.asris.csiro.au/ASRISApi/api/APSIM/getApsoil) provided by the Australian Soil Resource Information System (ASRIS) as described by Thomas et al. (2019). To initialize forecast simulations, each simulation was run over 5 years preceding the forecasting period, referred to as the 'spin-up' period.

\subsection{GGTactical - deploying GrassGro ${ }^{\mathrm{TM}}$ for flexible batch processing of grazing simulations}

The GrassGro ${ }^{\mathrm{TM}}$ software application is normally distributed as a Microsoft Windows desktop application. For this project the core models, which reside in dynamic linked libraries, were hosted by a new command line interface program called GGTactical. The core models and GGTactical, written in Object Pascal, were recompiled for use on either Windows or 64-bit Linux systems.

GGTactical has incorporated features from the desktop application that include;

1. Ability to run an historical simulation

2. Ability to run a tactical or seasonal forecast simulation

3. An output data reporting system that aggregates simulation outputs

Extra features it incorporated include:

1. Fully configured from command line arguments

2. Access to weather data web services

3. Access to soil information via the ASRIS web service

4. APSOIL information can be used from data files to get soil information

By combining these streams of simulation data, a historical context for current and likely upcoming seasonal conditions can be determined. The seasonal outlook in GGTactical uses climatology over a period of usually 20-30 years of weather data for the specified location, determined to represent enough variability in climate to adequately explain future scenarios. The diagrams below illustrate the seasonal outlook process (Figures 1a, b). The 'spinup' period sets the initial state of the farm system. From that point the climatology can provide a good representation of the potential future variability, assuming long term climatic conditions are stable.

a.

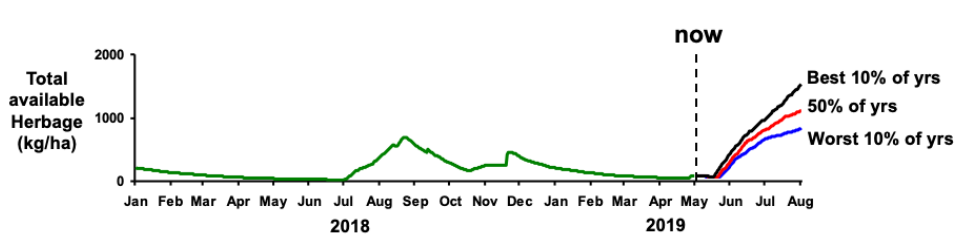

b.

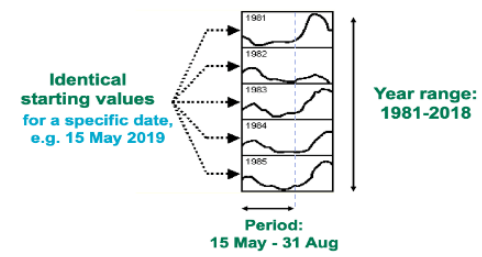

Figure 1. a) Percentile forecasts $\left(10^{\text {th }}, 50^{\text {th }}\right.$ and $\left.90^{\text {th }}\right)$ of total available pasture herbage $(\mathrm{kg} / \mathrm{ha})$, following simulated results for the preceding season. b) The same initial starting condition are used to initialize inseason simulations across many years (e.g. 1981 - 2018), which generate the probabilistic forecast scenarios.

\subsection{Pasture API - dynamic platform for connecting the pasture simulation engine with data streams}

Pasture API is a cloud hosted solution that exposes a subset of core features available in the GrassGro ${ }^{\mathrm{TM}}$ engine. It is enabled by the Senaps platform and manifests itself within the platform as a Senaps operator (Coombe et al. 2017) (Figure 2). A Senaps operator is conceptually a unit of execution and can be linked directly to live and static data sources when embedded within a Senaps workflow. A workflow can be run on 
demand or on a schedule. Each GrassGro ${ }^{\mathrm{TM}}$ input and output (exposed as ports of an operator) is mapped internally to the underlying GGTactical command line interface which allows the underlying model to be connected directly to existing near-realtime Senaps data sources. Senaps data sources used within the context of Pasture API are typically static documents (i.e. parameter files), datastreams, and/or grids (i.e. netcdf data); data sources include SILO climate and weather data but could also be extended to on farm weather station data for example.

Prior to developing Pasture API, the typical way interact with GrassGro ${ }^{\mathrm{TM}}$ was to install the standalone desktop application and perform analysis locally on the host machine (https://grazplan.csiro.au/grassgro/). The desktop application, which has been commercially available since 1997, is a feature rich and highly customizable decision support tool and has been continuously developed to improve its capacity to support both research and commercial applications. However, in an ever-changing digital landscape, it was necessary to build the Pasture API to achieve the benefits of exposing GrassGro ${ }^{\mathrm{TM}}$ features to the broader digital ecosystem that can benefit from using these models as back-end services.

Application developers wanting to explore and use this API can prepare and submit their own Senaps workflow and choose to expose the raw model results from within applications by consuming the data via the Senaps APIs or they can optionally perform further analysis by chaining results to downstream Senaps workflows. An example of a live application installing site specific workflows and consuming the raw results has been demonstrated in the Pasture Tracker interface (see section 2.5).

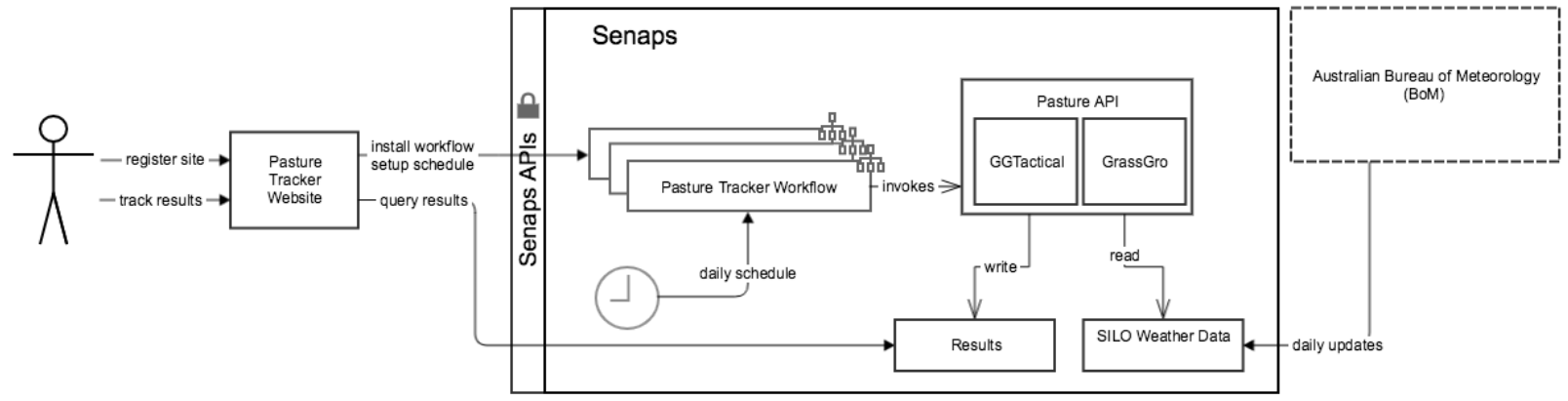

Figure 2. Senaps workflows are a way of interacting with the 'Pasture API' operator linking in historic weather observations and customisations specific to their site location.

\section{4. 'Pasture Tracker' - demonstration interface}

Pasture tracker is a prototype web application designed to demonstrate the Pasture API application to livestock producers, agricultural information providers, research and development corporations and other interested parties in the agricultural industry. The pasture tracker can be accessed using a password protected login, and provides a simplified, focussed interface for easily generating pasture forecasts based on a location and farming system that best represents their current enterprise. Once a new site is registered with Pasture Tracker, it is scheduled to run regularly (every day) behind the scenes allowing users to revisit the application and monitor progress on how their original forecast is tracking. Summary results and interactive charts are displayed on the website and intended to be available anywhere with an internet browser (Figure 3). The underlying GrassGro ${ }^{\mathrm{TM}}$ engine used to generate results supports a number of biophysical simulation outputs and for this initial version of Pasture Tracker, 'Projected green available herbage' was selected as a starting point to demonstrate example simulation results. In the future, other report types such as Plant Available Water, Maintenance supplement intake, Adult Ewe Condition Score, Ground Cover, Lamb Liveweight are planned to be included in upcoming versions in the form of charts and tables. The web application itself was created using a javascript framework and interfaces directly with Senaps APIs and requires no intermediate service layer or 'back-end'. All simulation results are generated via Pasture API (see section 2.3) using the GrassGro $^{\mathrm{TM}}$ engine, and authentication, authorisation, and supporting queries are handled by Senaps (See Figure 2).

\section{SENSITIVITY ANALYSIS OF SIMULATION INPUT STREAMS}

As described previously, the Pasture API application is designed so that input and parameter data may be ingested from various sources, including databases, libraries, field measurements and sensors. In some cases these data streams offer alternate corresponding data streams, providing the opportunity to select and compare between data sources for their accuracy and reliability. In addition, we emphasised the use of generic data streams to enable the application to be used across a wider geospatial area and reducing the need for local data 
streams, which can be an expensive and time consuming to produce. This raises the question as to the effects of using various data streams on the simulation scenarios, and how to target resources to ensure that the value of any improvements to data streams are optimised. To address this, we conducted sensitivity analyses comparing the effects of different soil, pasture and spin-up data sources on the simulation results and report on the effects on pasture net primary production (NPP) simulated by GrassGro ${ }^{\mathrm{TM}}$.

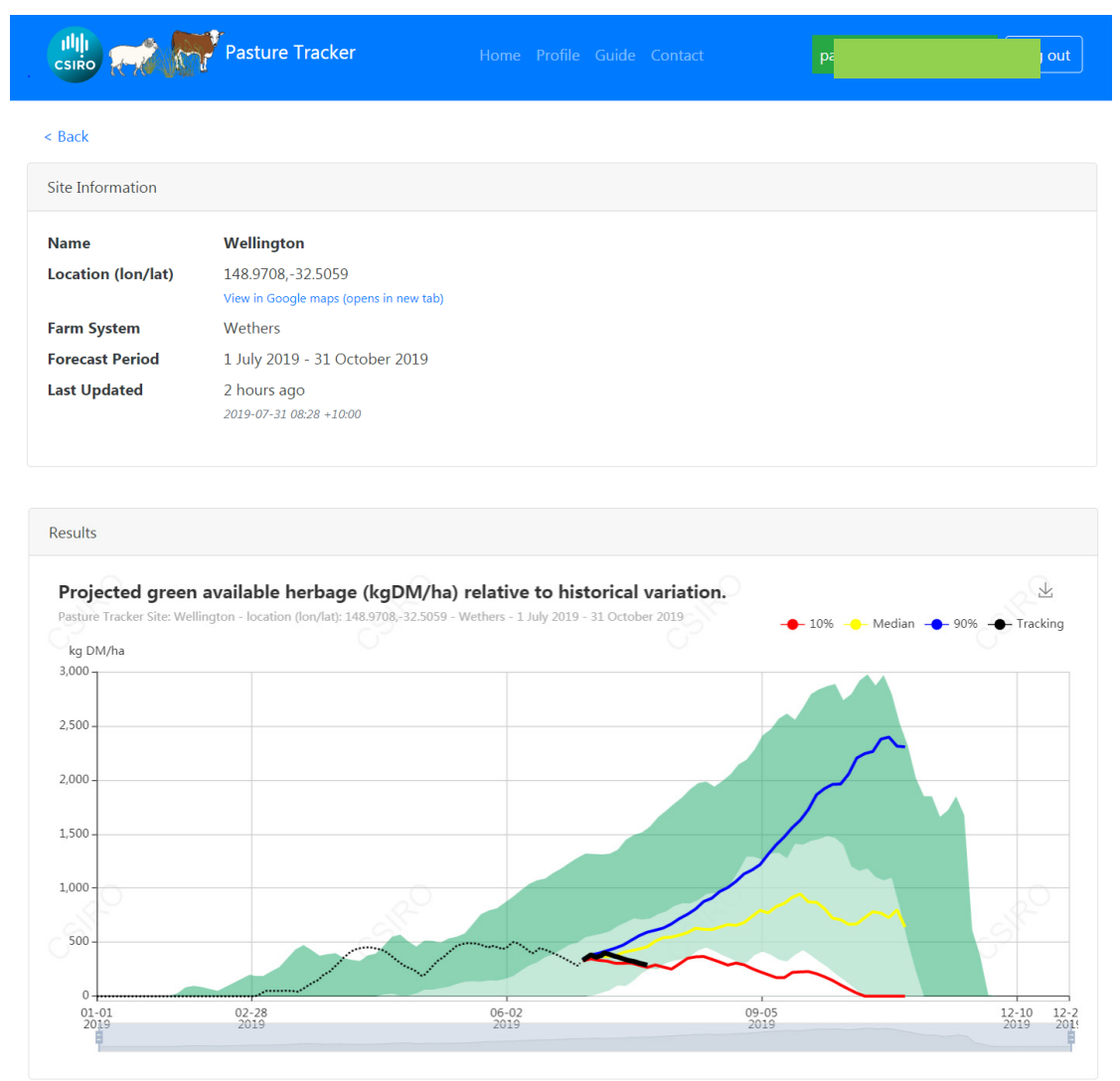

Figure 3. Pasture Tracker - Website interface with Pasture API. Users can create new sites via the dashboard and revisit the application to see how their registered site is tracking. The chart shown displays historic, current season, and probabilistic forecasts of green available herbage $(\mathrm{kgDM} / \mathrm{ha})$.

Using the GGTactical application we ran scenarios for 14 locations in NSW, Australia. The livestock enterprises at these locations were based on existing GrassGro ${ }^{\mathrm{TM}}$ libraries that have been developed and used by local consultants to provide advice on seasonal outlooks and tactical decision making by livestock managers. The structure of the livestock enterprises (e.g. animal breeds, stocking rate, reproduction and sale rules) differed across the sites, and we did not change the basic structure of any of the enterprises for the sensitivity analyses. The sensitivity analyses performed are described in Table 1 . All combinations of these sensitivity analysis conditions were tested based on monthly forecast outputs, a total of 112896 simulations (14 locations x 2 pasture species combinations $\mathrm{x} 2$ soil characterizations $\mathrm{x} 2$ forecast seasons $\mathrm{x} 2$ forecast initialisation (spin-up) years x 3 biomass initialisation adjustments x 28 forecast years x 6 forecast months). Analysis of variance, including interactions, was conducted for NPP using R version 3.6.0.

Comparison of the two different sources of pasture and soil inputs shows that effects on pasture growth (NPP) were influenced by interactions with location (pasture $\mathrm{x}$ soil $\mathrm{x}$ location; $\mathrm{F}=9.96, \mathrm{P}<0.001$; Figure 4). Overall, average monthly NPP of locally described soils and pastures was $17 \%$ higher than those from our generic soil and pasture database (716 v $713 \mathrm{~kg} /$ ha.month). However, there was also an effect of season (pasture $\mathrm{x}$ soil $\mathrm{x}$ month; $\mathrm{F}=2.73, \mathrm{P}=0.002$ ). During the winter months locally described pastures grew $19 \%$ higher than MLA feedbase audit-derived pastures, while the source of soil input data had no effect. Conversely, during spring NPP on locally described soils was c. 10\% higher than for ASRIS-derived soils (1099 v $1005 \mathrm{~kg} / \mathrm{ha}$.month), while the growth of locally described pastures was slightly lower than for the MLA feedbase audit-derived pastures (1077 v $1027 \mathrm{~kg} /$ ha.month). Our results suggest that during Winter simulations will be most sensitive to the plant model parameters, while in Spring and Summer soil characterisation will be more important for these grazing systems models. 
Table 1. Combinations of input data and parameters used in the sensitivity analyses.

\begin{tabular}{lll}
\hline Data or parameter & Sensitivity analyses combinations & \\
\hline $\begin{array}{l}\text { Pasture species } \\
\text { combination }\end{array}$ & $\begin{array}{l}\text { Locally defined by farmers and } \\
\text { consultants }\end{array}$ & $\begin{array}{l}\text { Based on an MLA feedbase audit report } \\
\text { (Donald, 2012) }\end{array}$ \\
Soil characterization & $\begin{array}{l}\text { Locally defined from field soil } \\
\text { surveys }\end{array}$ & $\begin{array}{l}\text { Based on Australian Soil Resource } \\
\text { Information System (ASRIS) (Thomas et } \\
\text { al. 2019) }\end{array}$ \\
$\begin{array}{l}\text { Forecast seasons } \\
\text { Forecast initialisation } \\
\text { conditions }\end{array}$ & $\begin{array}{l}\text { Initialisation in an above-average } \\
\text { rainfall season (year selected was } \\
2011,75^{\text {th }} \text { percentile) }\end{array}$ & $\begin{array}{l}\text { September-February } \\
\text { Initialisation in a below-average rainfall } \\
\text { season (year selected was 2018, 15 } \\
\text { percentile) }\end{array}$ \\
\hline
\end{tabular}

a.

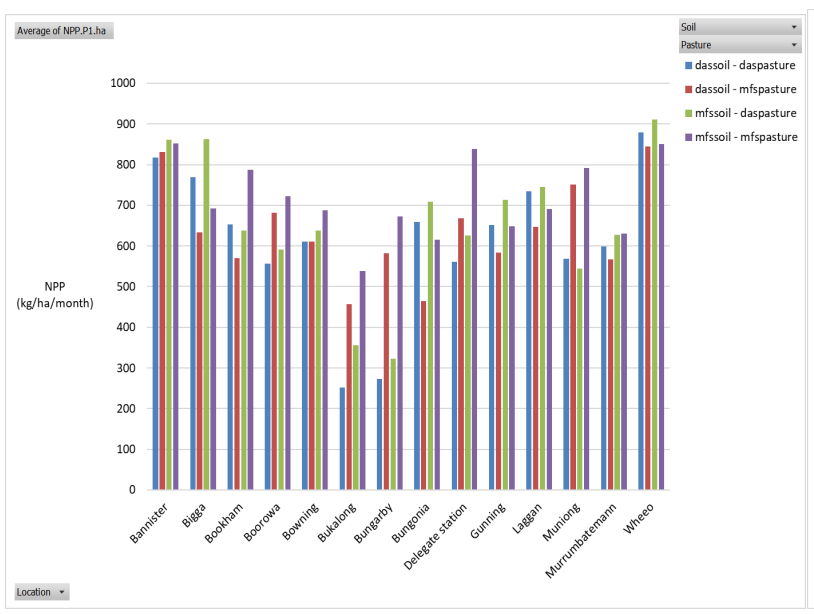

b.

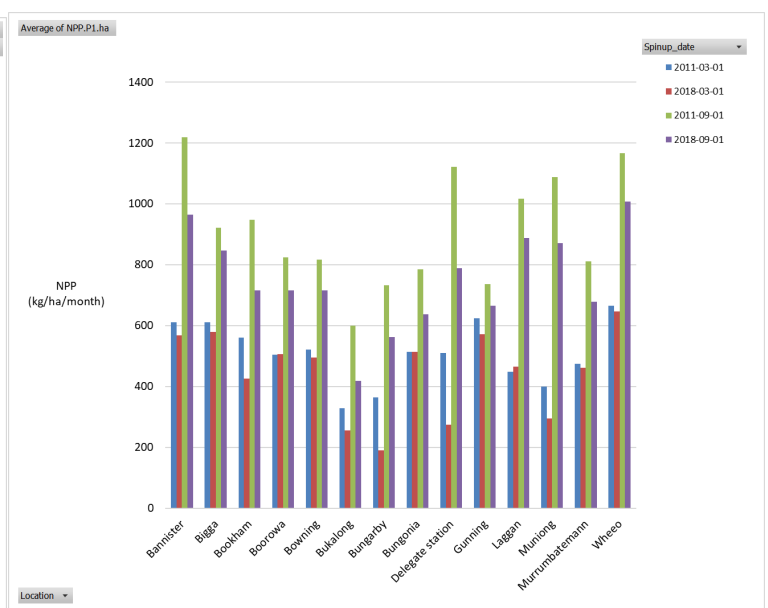

Figure 4. a) Mean monthly pasture production (NPP) for simulations with pastures and soils characterised locally, or from ASRIS or MLA feedbase audit databases. b) Mean monthly pasture production (NPP) for simulations with pastures and soils characterised locally, or from ASRIS or MLA feedbase audit databases.

The second part of the sensitivity analysis was to understand the effects of simulation spin-up conditions on subsequent forecast outcomes. The forecasting initialisation through the simulation spin-up was affected by the preceding seasonal conditions, as might be expected. That is, simulated pasture production (NPP) was lower when the spin-up preceding the forecasts was carried out in dry year compared with a wet year. There was a significant spin-up year $\mathrm{x}$ month interaction, where the difference in forecast NPP between simulation spin-up in a dry and wet was greater for the Spring spin-up compared with the Autumn spin-up. However, there was also a location $\mathrm{x}$ spin-up year (wet or dry) $\mathrm{x}$ month interaction as the effect of spin-up year had a more even effect for the Spring spin-up, whereas the effect varied more by location for the Autumn spin-up, with no effect at some locations (location x spin-up year (wet/dry) x month; $\mathrm{F}=10.58, \mathrm{P}<0.001$ )

\section{DISCUSSION AND CONCLUSION}

This paper described the development of Pasture API, a digital platform to support the development of feedbase management tools for the livestock industry of southern Australia. To achieve this, Pasture API provides an interface that connects a range of streams of input data with the GrassGro ${ }^{\mathrm{TM}}$ simulation modelling engine. Through automation of scenario development and processing, we were able to provide pasture forecast information for the current season at any location across the agricultural region of southern Australia, in the context of historical climatic conditions for the location. The potential for improving the pasture forecasts through incorporating seasonal climate model data (e.g. the Bureau of Meteorology's ACCESS-S1; Hudson et al. 2017) will also be evaluated across key representative grazing regions. The use of biophysical process modelling to represent livestock enterprises, allowed a high level of flexibility in the integration of multiple alternative streams as well as a wide range of potential input data types, of which we compared several in a sensitivity analysis. 
The proliferation of agricultural sensors has produced an equally large volume of proximal and remote sensed data. In many cases the sensor data could be highly valuable if it was stored and integrated within an analytical framework and then available for use in decision making. Rather, farm sensor data is currently used simply to generate alerts or for intermittent checking of near real-time measurements and then effectively discarded. In contrast, the Pasture API platform provides a framework for data storage and integration, and so is an example of how multiple data streams can be used to inform simulated scenarios and applied in tactical decision making.

In the sensitivity analysis, effects of different data streams that we had access to deploy in the Pasture API platform were compared. In most cases, generic (not locally sourced) soil and pasture data streams allowed a reasonable seasonal forecast that did not differ substantially from locally sourced data. However, at some locations where the pasture species combinations differed widely in their growth potential, the differences in forecast pasture production were large. Based on these results we suggest that it would be better to provide end users the ability to select the most relevant species composition for their area of interest and either select or input soil data that is locally relevant. Alternatively, further work to improve the accuracy of the generic input databases could be considered. Representing accurately pasture composition will likely have a major influence on the livestock enterprise through the amount and quality of feed from the simulated pastures. Validation studies where field data is collected from livestock enterprises would be beneficial to test further the performance of the simulation platform in relation to the various streams of input data.

In the Pasture API project we demonstrate the potential to create information for decision support in a livestock enterprise based on current and archived data streams and databases, using scenario modelling as an analytical framework. This implementation underpins efforts to transform agricultural forecasting from a static and locally deployed activity into a highly scalable and responsive system, producing readily consumable information for a range of decision support applications.

\section{ACKNOWLEDGMENTS}

This project was funded by CSIRO Agriculture and Food. We thank Phil Graham, Doug Alcock and the Monaro Farming Systems producer group for the livestock systems, soil and pasture data needed for the sensitivity analyses. We thank Dr Hayley Norman and Dr Michael Robertson for helpful suggestions on the manuscript.

\section{REFERENCES}

Bell, L.W., Robertson, M.J., Revell, D.K., Lilley, J.M., Moore, A.M. (2008) Approaches for assessing some attributes of feed-base systems in mixed farming enterprises. Australian Journal of Experimental Agriculture. 48, 789-798.

M. Coombe, P. Neumeyer, J. Pasanen, C. Peters, C. Sharman, and P. Taylor. 2017. Senaps: A platform for integrating time-series with modelling systems. In: Syme, G., Hatton MacDonald, D., Fulton, B., Piantadosi, J. (Eds.). 22nd International Congress on Modelling and Simulation (MODSIM), Hobart, Australia, 3-8 December 2017. pp. 438-444.

Donald, G. (2012). Analysis of Feed-base Audit. Final Report for project B.PAS.0297. Meat and Livestock Australia, North Sydney, NSW.

Herrmann, N.I., Zurcher, E.J., (2011). Designing decision support systems for factorial analyses. In: Chan, F., Marinova, D., Anderssen, R.S. (Eds.), 19th International Congress on Modelling and Simulation. Perth, Australia, 12-16 December 2011. pp. 1209-1215.

Hudson, D., Alves, O., Hendon, H., Lim, E.-P., Liu, G., Luo, J.-J., MacLachlan, C., Marshall, A., Shi, L., Wang, G., Wedd, R., Young, G., Zhao, M., Zhou, X. (2017) ACCESS-S1: The new Bureau of Meteorology multi-week to seasonal prediction system. Journal of Southern Hemisphere Earth Systems Science. 67, 132159.

Jeffrey, S.J., Carter, J.O., Moodie, K.M. and Beswick A.R. (2001) Using spatial interpolation to construct a comprehensive archive of Australian climate data. Environmental Modelling \& Software. 16, 309-330.

Mata, G., Henry, D.A., Gherardi, S.G., Smith, R.C.G. (2004) Pastures from Space - introduction to the technology on farm. Animal Production in Australia. 25, 281.

Moore AD, Donnelly JR, Freer M (1997). GRAZPLAN: decision support systems for Australian enterprises. III. Pasture growth and soil moisture submodels, and the GrassGro DSS. Agricultural Systems. 55, 535582.

Thomas, D.T., Zurcher, E.J., Mata, G., Herrmann, N.I., Henry, D.A. (2019) An estimate of carrying capacity of land for ruminant livestock production across southern Australia, using gridded batch simulation modelling. In: Proceedings of the 16th Australian Agronomy Conference, Wagga Wagga, NSW, Australia, 25-29 August 2019. In Press. 